C Revista de Matemática: TeOría y ApliCACiones 2021 28(1) : 125-142

CIMPA - UCR ISSN: 1409-2433 (PRINT), 2215-3373 (ONLINE)

DOI: https://doi.org/10.15517/rmta.v28i1.41890

ESTUDIO COMPARATIVO ENTRE LA VELOCIDAD Y LA DENSIDAD EN MODELOS MACROSCÓPICOS DEL TRÁFICO VEHICULAR INCORPORANDO LA VARIABLE ESPACIAL EN LA VELOCIDAD

\author{
A COMPARATIVE STUDY ON SPEED AND \\ DENSITY IN MACROSCOPIC MODELS OF \\ VEHICULAR TRAFFIC ADDING THE SPATIAL \\ VARIABLE ON SPEED
}

José Mauricio AlvarengA-RodríGUeZ*

Received:02/Jun/2020; Revised:07/Aug/2020; Accepted: 30/Oct/2020

Revista de Matemática: Teoría y Aplicaciones is licensed under a Creative Commons Attribution-NonCommercial-ShareAlike 4.0 International License. http://creativecommons.org/licenses/by-nc-sa/4.0/

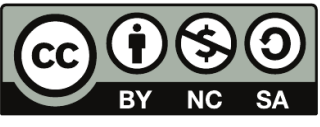

*Universidad Nacional Autónoma de Honduras, Departamento Matemática Aplicada, Tegucigalpa, Honduras. E-Mail: jose.alvarenga@unah.edu.hn 


\title{
Resumen
}

El presente trabajo tiene como propósito comparar las relaciones clásicas entre densidad y velocidad en el contexto de los modelos continuos del tráfico vehicular, integrando una dependencia lineal de la variable espacial. Para llevar a cabo dicha tarea se usó la base de datos del proyecto NGSIM, específicamente, la información recolectada en la carretera I-80. Además, para hacer una comparación equilibrada también se estimaron los parámetros de las relaciones clásicas sin la modificación lineal de la variable espacial.

Palabras clave: velocidad; flujo vehicular; densidad vehicular.

\begin{abstract}
The present work aims to compare the classic relations between density and speed in the context of continuous models of vehicular traffic, integrating a linear dependence of the spatial variable. Database from NGSIM was used in order to accomplish such task, specifically that belonging to I-80 highway. Furthermore, to make a balanced comparison, the parameters of the classical relations were also estimated without the linear modification of the spatial variable.
\end{abstract}

Keywords: speed; vehicle flow; vehicle density.

Mathematics Subject Classification: 93A30.

\section{Recolección de la información}

En este trabajo se utilizó parte de una de las mejores bases de datos existentes en información del tráfico vehicular. El proyecto NGSIM (Next Generation Simulation Vehicle Trajectories and Supporting Data) contiene el registro de cientos de miles de datos asociados a distintos vehículos observados en ciertos sectores de carreteras y calles de Estados Unidos. Específicamente dicha base de datos recogió de cada vehículo observado, la posición, velocidad, tamaño, aceleración y distancia al vehículo enfrente de este, en diferentes instantes de tiempo. Es por la disponibilidad tan específica que posee este conjunto de datos, que es posible aplicarlos al estudio de los modelos continuos del tráfico vehicular.

Como se puede consultar en [10], el proyecto se realizó en la ruta federal 101, el bulevar de Lankershim, la interestatal 80, en el estado de California y la calle Peachtree en Atlanta. En este artículo se usó la información correspondiente a los datos recolectados en la interestatal 80 .

Inicialmente se descargaron los datos del proyecto de la página web [10], posteriormente, se desarrolló un código de programación para fragmentar y manipular estos datos, puesto que la cantidad de información es considerablemente 
grande, y finalmente se procedió a la calibración de los modelos. En [1] se puede observar una reconstrucción de la dinámica del proyecto NGSIM en la interestatal 80 durante los primeros 15 minutos de captación de datos hecha por el autor de este artículo.

\section{Modelos macroscópicos del tráfico vehicular}

Existen un conjunto de modelos denominados continuos para el tráfico vehicular que tienen su origen en el artículo [7], aquí se conjetura una relación de dependencia entre velocidad y densidad vehicular, esta relación dio lugar a dos amplias ramas que se identifican por los modelos microscópicos y macroscópicos del tráfico vehicular.

Este estudio está orientado a los modelos macroscópicos. Las magnitudes centrales en todo este trabajo son las siguientes:

1. La densidad vehicular, que se mide en términos del número de carros por unidad de longitud, se representa por la letra $\rho$.

2. La velocidad del tráfico vehicular, que se mide en unidades de longitud por unidad de tiempo, se denota por $v$.

Tabla 1: Modelos macroscópicos clásicos.

\begin{tabular}{c|cc}
\hline \hline Modelo & Dependencia & Parámetros \\
\hline Greenshields [7] & $v=v_{\max }\left(1-\rho / \rho_{\max }\right)$ & {$\left[v_{\max }, \rho_{\max }\right]$} \\
Greenberg [6] & $v=v_{c} \log \left(\rho_{\max } / \rho\right)$ & {$\left[v_{c}, \rho_{\max }\right]$} \\
Underwood [11] & $v=v_{\max } e^{-\rho / \rho_{c}}$ & {$\left[v_{\max }, \rho_{c}\right]$} \\
Drake [3] & $v=v_{\max } \exp \left(-\rho^{2} /\left(2 \rho_{c}^{2}\right)\right)$ & {$\left[v_{\max }, \rho_{c}\right]$} \\
Drew[4] & $v=v_{\max }\left(1-\left(\rho / \rho_{\max }\right)^{1 / 2}\right)$ & {$\left[v_{\max }, k_{j}\right]$} \\
Pipes[9] & $v=v_{\max }\left(1-\rho / \rho_{\max }\right)^{2}$ & {$\left[v_{\max }, k_{j}\right]$} \\
Nicholas[8] & $v=v_{\max }\left(\rho_{\max }^{n}-\rho^{n}\right) /\left(\rho_{\max }^{n}+m \rho^{n}\right)$ & {$\left[v_{\max }, k_{j}, n, m\right]$} \\
\hline \hline
\end{tabular}

En [7] se conjeturó que la velocidad dependía linealmente de la densidad. Otros autores encontraron diferentes tipos de dependencias en este sentido, la Tabla 1 contiene un conjunto de dependencias clásicas de la velocidad en función de la densidad. 
En el desarrollo de los modelos macroscópicos se han considerado algunos modelos en los que se incorpora, además de la densidad, la dependencia de la variable espacial, como un ejemplo considérese el trabajo [12] donde se hace un estudio teórico bajo este supuesto.

En este artículo se considerará una dependencia de la siguiente forma:

$$
v(\rho, x)=(a x+b) g(\rho) .
$$

La ecuación (1) supone dos ventajas, una es que la dependencia no aumenta demasiado la complejidad, al ser lineal en la variable espacial, y la dependencia puede captar sensibilidades a aspectos propios de la carretera de estudio y no de la densidad únicamente. Tomando esto último en cuenta, en la Tabla 2 se observa esta modificación en los modelos clásicos.

Tabla 2: Modelos macroscópicos clásicos, incorporando una dependencia lineal en la variable espacial.

\begin{tabular}{c|cc}
\hline \hline Modelo & Dependencia & Parámetros \\
\hline Greenshields & $v=(a x+b)\left(1-\rho / \rho_{\max }\right)$ & {$\left[a, b, \rho_{\max }\right]$} \\
Greenberg & $v=(a x+b) \log \left(\rho_{\max } / \rho\right)$ & {$\left[a, b, \rho_{\max }\right]$} \\
Underwood & $v=(a x+b) e^{-\rho / \rho_{c}}$ & {$\left[a, b, \rho_{c}\right]$} \\
Drake & $v=(a x+b) \exp \left(-\rho^{2} /\left(2 \rho_{c}^{2}\right)\right)$ & {$\left[a, b, \rho_{c}\right]$} \\
Drew & $v=(a x+b)\left(1-\left(\rho / \rho_{\max }\right)^{1 / 2}\right)$ & {$\left[a, b, k_{j}\right]$} \\
Pipes & $v=(a x+b)\left(1-\rho / \rho_{\max }\right)^{2}$ & {$\left[a, b, k_{j}\right]$} \\
Nicholas & $v=(a x+b)\left(\rho_{\max }^{n}-\rho^{n}\right) /\left(\rho_{\max }^{n}+m \rho^{n}\right)$ & {$\left[a, b, k_{j}, n, m\right]$} \\
\hline \hline
\end{tabular}

\section{Exploración de la base de datos}

En esta parte se describirán algunas características de los datos utilizados. Las tres cantidades más relevantes para este estudio, corresponden con la velocidad, la densidad y el flujo. En la Figura 1 se observan los histogramas de las densidades en los diferentes carriles.

Con la Figura 1 puede conjeturarse el valor de la densidad máxima en cada uno de los carriles. Por ejemplo, en el carril 5 se observa que la densidad máxima (como valor promedio) se ubica entre 80 y 100 vehículos por kilómetro.

De forma similar, se han obtenido los histogramas correspondientes a la velocidad y el flujo en las Figuras 2 y 3 . Nuevamente se puede conjeturar un rango de valores máximos, tanto para la velocidad como para el flujo. 

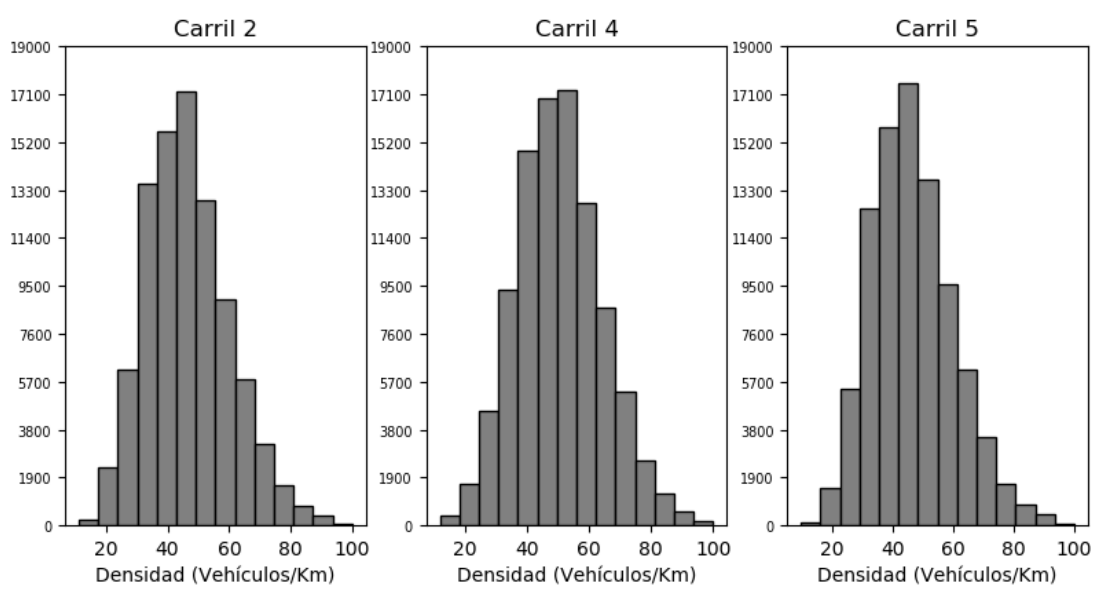

Figura 1: Frecuencias de las densidades en los diferentes carriles.
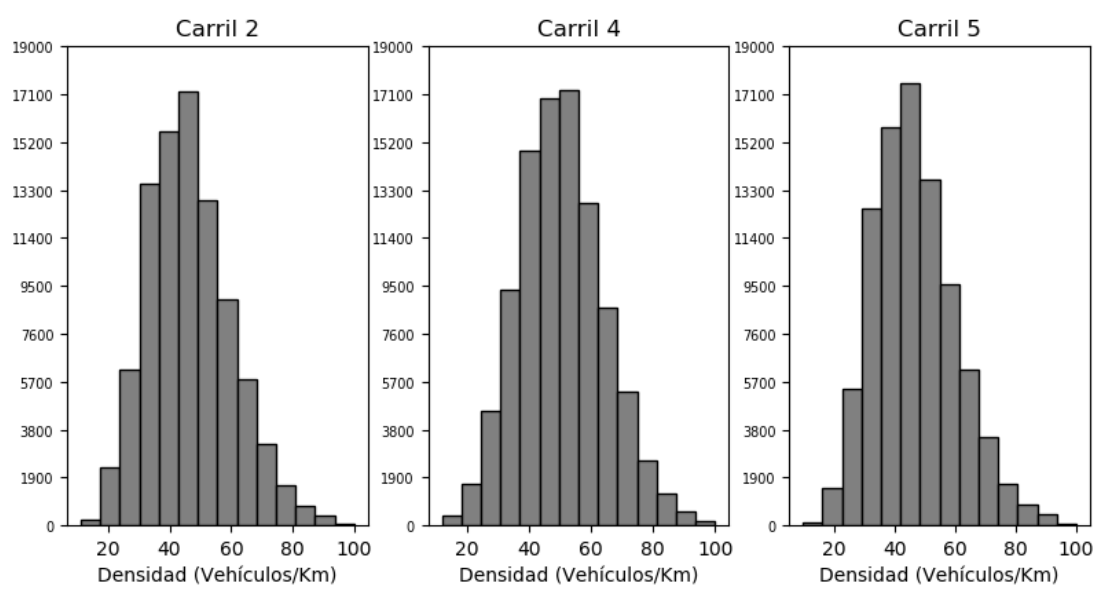

Figura 2: Frecuencias de las velocidades en los diferentes carriles. 

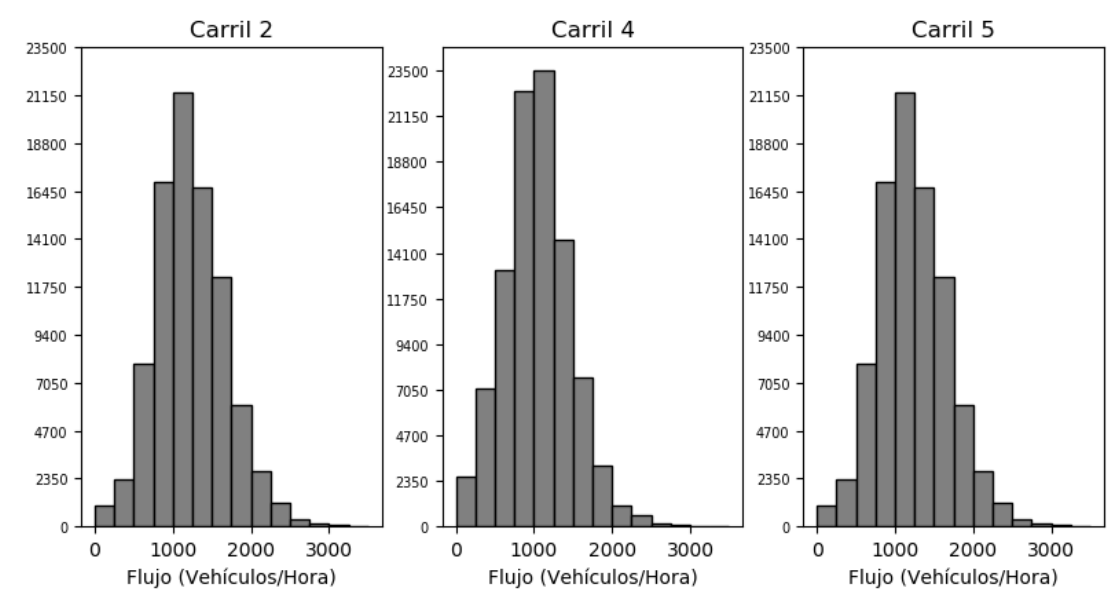

Figura 3: Frecuencias de los flujos en los diferentes carriles.

Utilizando la información que se transparenta en los histogramas, se han estimado los posibles valores para los siguientes parámetros promedios:

1. La densidad máxima, denotada por $\rho_{\max }$.

2. La velocidad promedio, denotada por $v_{\max }$.

3. La densidad óptima, denotada por $\rho_{c}$. Este parámetro se entiende como la densidad en que se alcanza el flujo máximo.

Haciendo uso de una técnica de remuestreo (bootstrap) se han estimado los parámetros anteriores con ayuda de los valores máximos de la densidad, velocidad y flujo de la base de datos proveniente del estudio hecho en la interestatal 80 por parte del proyecto NGSIM. Las estimaciones y los intervalos de confianza $(\alpha=0.05)$ de los valores promedios de la densidad, velocidad y flujo máximo se observan en la Tabla 3.

Tabla 3: Estimación de los parámetros $\rho_{\max }, v_{\max } \mathrm{y} \rho_{c}$. El encabezado I.C. indica el intervalo de confianza de la estimación del valor promedio.

\begin{tabular}{c|cccccc}
\hline \hline Carril & $\rho_{\max }$ & I.C. & $v_{\max }$ & I.C. & $\rho_{c}$ & I.C. \\
\hline 2 & 86.45 & $(86.14,86.74)$ & 43.83 & $(43.75,43.91)$ & 69 & $(68.18,69.8)$ \\
4 & 88.09 & $(87.61,88.5)$ & 42.43 & $(42.27,42.59)$ & 74.65 & $(72.88,76.31)$ \\
5 & 90.95 & $(90.49,91.4)$ & 45.31 & $(45.18,45.45)$ & 79.46 & $(76.82,81.96)$ \\
\hline \hline
\end{tabular}


Las estimaciones realizadas anteriormente serán utilizadas al final de este trabajo para contrastar con los parámetros encontrados, producto del ajuste de los modelos estudiados.

Para finalizar esta sección, se presenta en la Figura 4 un histograma bidimensional que evidencia la relación inversamente proporcional, existente entre la velocidad y la densidad.
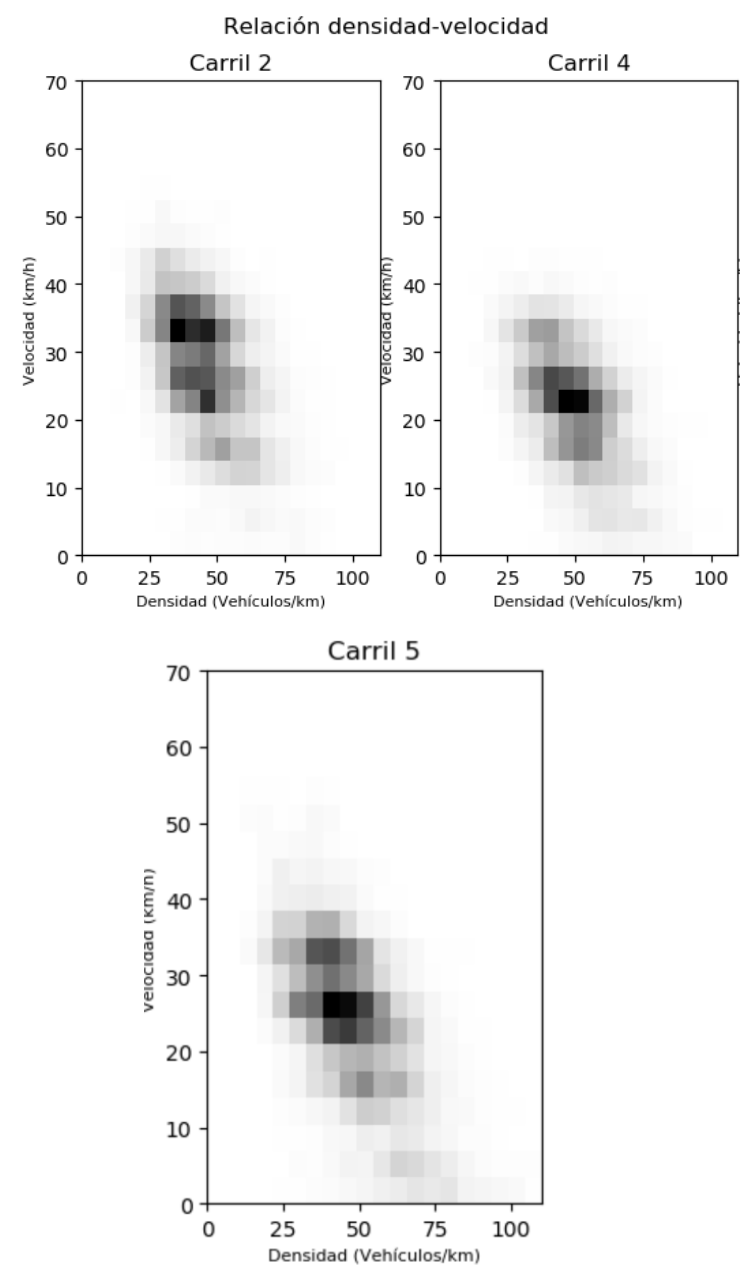

Figura 4: Frecuencias de las parejas de densidad y velocidad. Los puntos más oscuros indican frecuencias grandes. 


\section{Estimación de los parámetros asociados a los modelos macroscópicos}

Para estimar los parámetros, se siguió el procedimiento utilizado en [6], aquí se agruparon los datos de acuerdo a las densidades observadas, específicamente se utilizaron 15 clases, o intervalos donde se promediaron tales densidades. Para incorporar la variable espacial, se hizo una partición tanto en el espacio como en la densidad.

Siendo más precisos, supóngase que se tiene un conjunto de datos de la forma $\left(\rho_{j}, v_{j}, x_{j}\right)$ para $j=1, \cdots, n$. Asúmase que se conocen las densidades mínimas y máximas denotadas por $\rho_{\min } \mathrm{y} \rho_{\max }$, y las posiciones mínimas y máximas denotadas por $x_{\min } \mathrm{y} x_{\max }$.

Ahora considérense las siguientes particiones:

$$
\begin{gathered}
\rho_{\min }=P_{1}<P_{2}<\cdots<P_{N+1}=\rho_{\max }, \\
x_{\min }=X_{1}<X_{2}<\cdots<X_{M+1}=x_{\max } .
\end{gathered}
$$

Con lo anterior se pueden calcular los siguientes puntos promedios:

$$
\begin{aligned}
\hat{\rho}_{k l}= & \frac{\sum_{i=1}^{n} \rho_{i} \chi_{\left[P_{k}, P_{k+1}\right]}\left(\rho_{i}\right) \chi_{\left[X_{l}, X_{l+1}\right]}\left(x_{i}\right)}{\sum_{i=1}^{n} \chi_{\left[P_{k}, P_{k+1}\right]}\left(\rho_{i}\right) \chi_{\left[X_{l}, X_{l+1}\right]}\left(x_{i}\right)}, \\
\hat{x}_{k l}= & \frac{\sum_{i=1}^{n} x_{i} \chi_{\left[P_{k}, P_{k+1}\right]}\left(\rho_{i}\right) \chi_{\left[X_{l}, X_{l+1}\right]}\left(x_{i}\right)}{\sum_{i=1}^{n} \chi_{\left[P_{k}, P_{k+1}\right]}\left(\rho_{i}\right) \chi_{\left[X_{l}, X_{l+1}\right]}\left(x_{i}\right)}, \\
\hat{v}_{k l}= & \frac{\sum_{i=1}^{n} v_{i} \chi_{\left[P_{k}, P_{k+1}\right]}\left(\rho_{i}\right) \chi_{\left[X_{l}, X_{l+1}\right]}\left(x_{i}\right)}{\sum_{i=1}^{n} \chi_{\left[P_{k}, P_{k+1}\right]}\left(\rho_{i}\right) \chi_{\left[X_{l}, X_{l+1}\right]}\left(x_{i}\right)} .
\end{aligned}
$$

Para $k=1, \cdots, N, l=1, \cdots, M$ y donde $\chi$ es la función característica de la teoría de conjuntos. Son estos puntos promedios los que se usarán para calcular los parámetros deseados. Para hacer el cálculo de los parámetros se usó el método de mínimos cuadrados para problemas no lineales, tal método 
se encuentra implementado en la función least_square de la librería scipy en Python y fue usada en este trabajo.

Otro aspecto que se consideró, con el objetivo de enriquecer el estudio, fue tomar en cuenta la estimación de los parámetros en distintos carriles de la interestatal 80. Se tomaron tres carriles del centro de la interestatal, los etiquetados con los índices 2,4 y 5. En las Figuras 5, 6 y 7 pueden observarse las estimaciones hechas en los carriles 2,4 y 5 .

Un tipo de gráfico útil para hacer una comparación rápida del ajuste de parámetros, se puede encontrar en los gráficos de caja y bigote de los errores de las estimaciones en la Figura 8. Los errores se calcularon tomando la diferencia entre $\hat{v}_{k l}$ y el modelo evaluado en los puntos $\hat{\rho}_{k l}$ y $\hat{x}_{k l}$. Para poder comprender claramente los gráficos en la Figura 8, se usa la siguiente notación:

1. M1: Modelo de Greenshields [7].

2. M1X: Modelo de Greenshields con modificación lineal espacial [7].

3. M2: Modelo de Greenberg [6].

4. M2X: Modelo de Greenberg con modificación lineal espacial [6].

5. M3: Modelos de Underwood [11].

6. M3X: Modelo de Underwood con modificación lineal espacial [11].

7. M4: Modelo de Drake [3].

8. M4X: Modelo de Drake con modificación lineal espacial [3].

9. M5: Modelo de Drew [4].

10. M5X: Modelo de Drew con modificación lineal espacial [4].

11. M6: Modelo de Munjal y Pipes [9].

12. M6X: Modelo de Munjal y Pipes con modificación lineal espacial [9].

13. M7: Modelo de MacNicholas [8].

14. M7X: Modelo de MacNicholas con modificación lineal espacial [8]. 


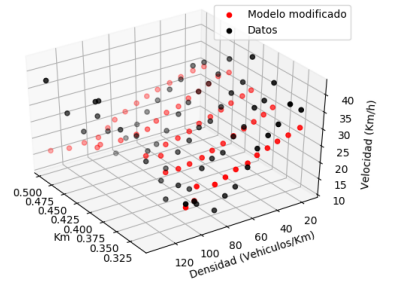

(a) Modelo de Greenshield en el carril 2.

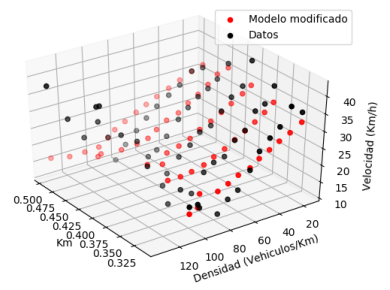

(c) Modelo de Underwood en el carril 2.

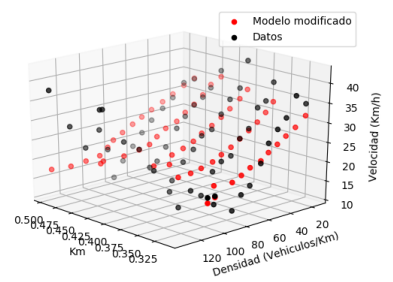

(e) Modelo de Drew en el carril 2.

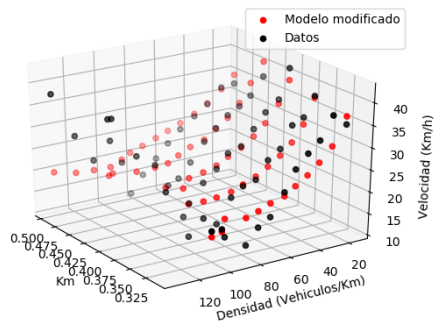

(g) Modelo de MacNicholas en el carril 2.

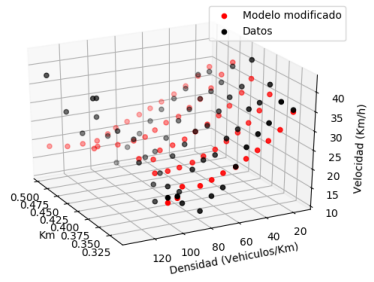

(b) Modelo de Greenberg en el carril 2.

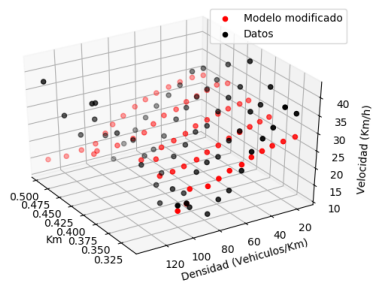

(d) Modelo de Drake en el carril 2.

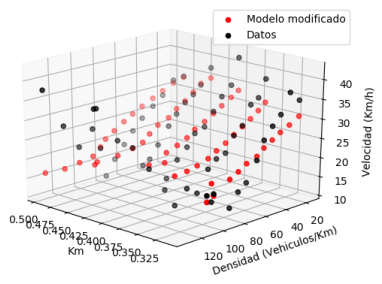

(f) Modelo de Pipes y Munjal en el carril 2.

Figura 5: Los puntos circulares representan los datos promedios y los triangulares las estimaciones. 


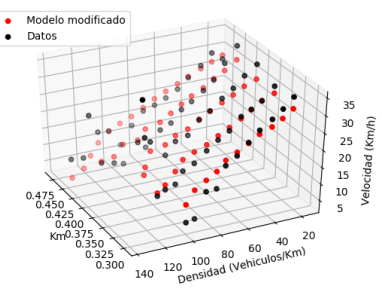

(a) Modelo de Greenshield en el carril 4.

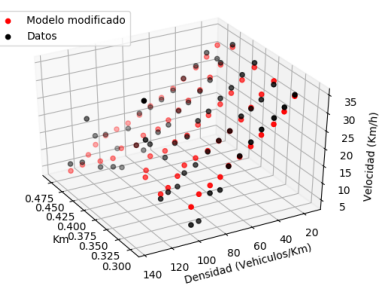

(c) Modelo de Underwood en el carril 4.

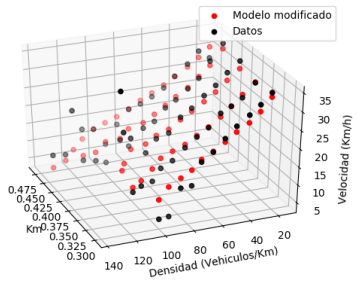

(e) Modelo de Drew en el carril 4.

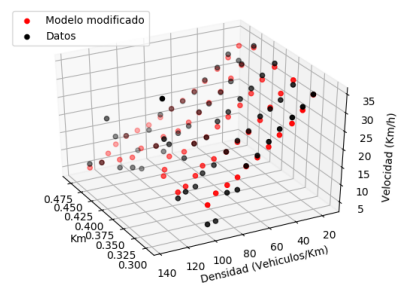

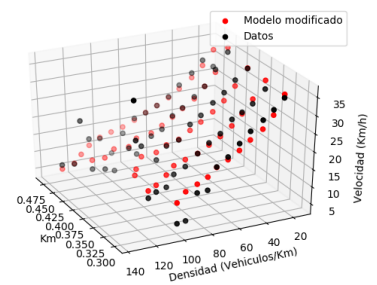

(b) Modelo de Greenberg en el carril 4.

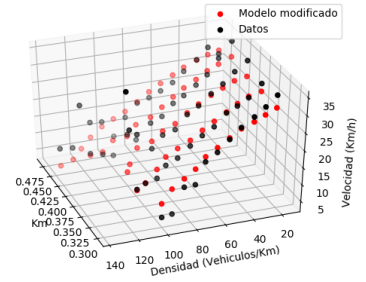

(d) Modelo de Drake en el carril 4.

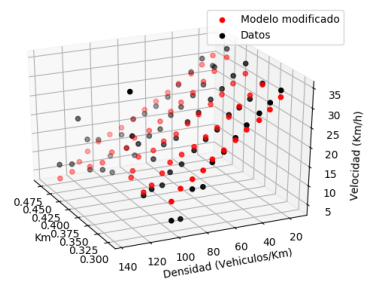

(f) Modelo de Pipes en el carril 4.

(g) Modelo de Nicholas en el carril 4.

Figura 6: Los puntos circulares representan los datos promedios y los triangulares las estimaciones. 


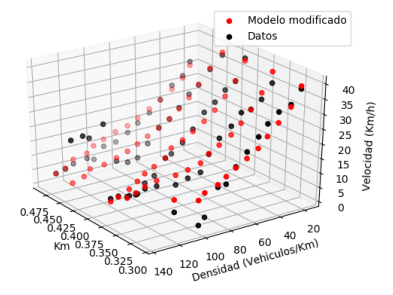

(a) Modelo de Greenshield en el carril 5.

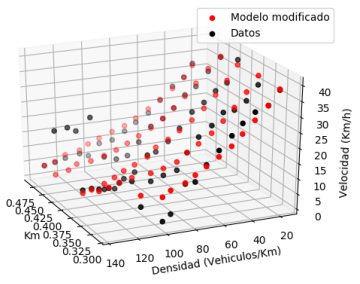

(c) Modelo de Underwood en el carril 5.

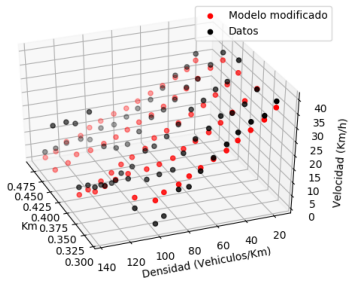

(e) Modelo de Drew en el carril 5.

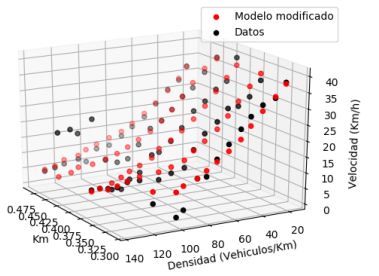

(g) Modelo de Nicholas en el carril 5.

Figura 7: Los puntos circulares representan los datos promedios y los triangulares las estimaciones.

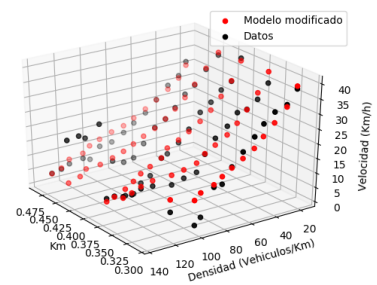

(b) Modelo de Greenberg en el carril 5.

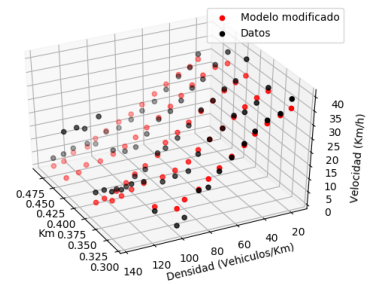

(d) Modelo de Drake en el carril 5.

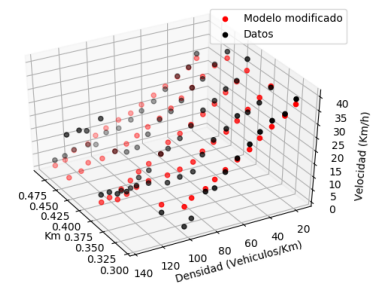

(f) Modelo de Pipes en el carril 5. 


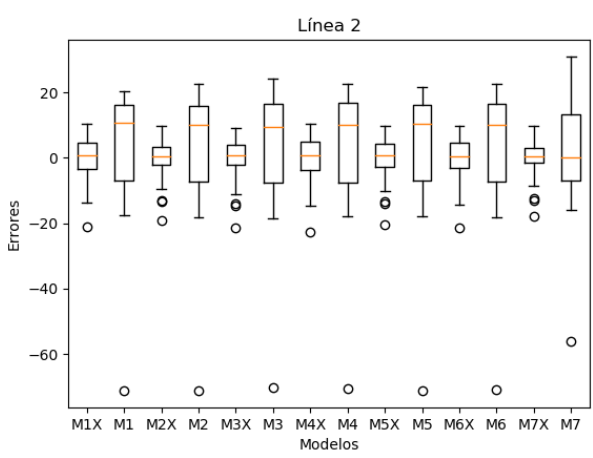

(a) Comparación de los errores en el carril 2.

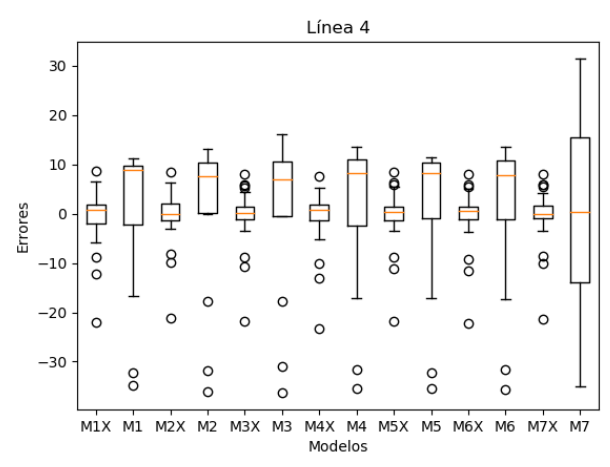

(b) Comparación de los errores en el carril 4.

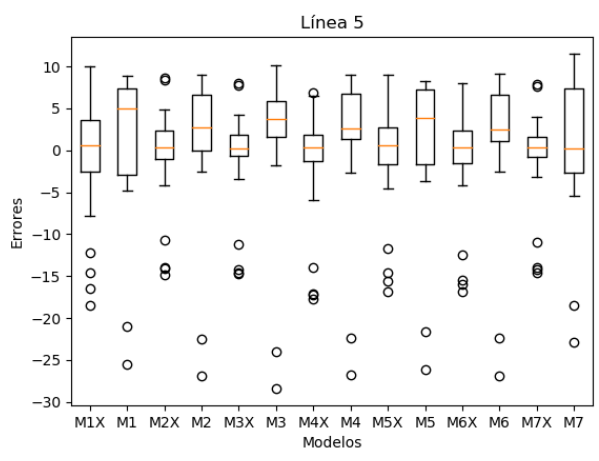

(c) Comparación de los errores en el carril 5.

Figura 8: Errores de las aproximaciones de los parámetros en cada uno de los carriles.

De la Figura 8 se pueden extraer las siguientes observaciones:

1. Se puede observar que en general en los modelos modificados se tiene una menor dispersión en los errores obtenidos.

2. También se puede notar que los gráficos son más simétricos en las cajas de los modelos modificados.

Otro gráfico de comparación se muestra en la Figura 9. Aquí se ha calculado el error estándar de cada una de las aproximaciones en cada carril. En la Figura 9 las líneas con marcas cuadradas representan a los modelos modificados. Rápidamente se puede apreciar que en general los errores estándares son considerablemente menores en los modelos modificados. 
Dejando de lado la comparación entre los modelos clásicos y los modificados, se evidencia que los tres mejores modelos modificados son el M2X, M3X y M7X, además se puede notar que son los modelos que tiene menor dispersión en la Figura 8.

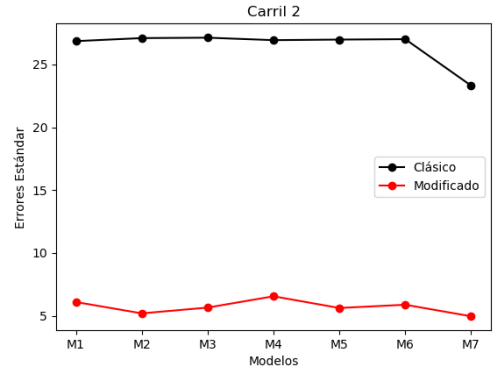

(a) Comparación de los errores en el carril 2.

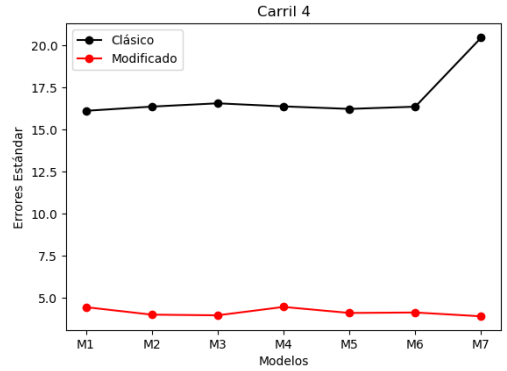

(b) Comparación de los errores en el carril 4.

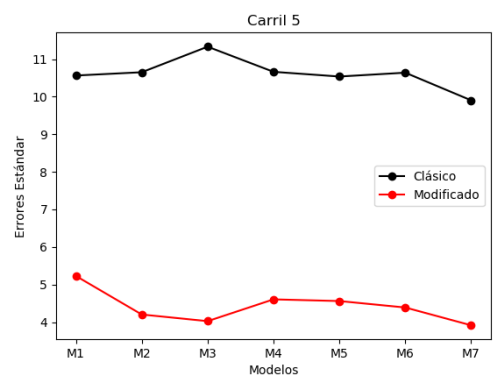

(c) Comparación de los errores en el carril 5.

Figura 9: Comparación de los errores estandar en cada uno de los carriles.

Para finalizar, en las Tablas 4, 5 y 6 se encuentran los parámetros encontrados en este trabajo. Los encabezados $a, b, v_{\max }, v_{c}, \rho_{\max }, \rho_{c}, n$ y $m$ hacen referencia a los parámetros de las Tablas 1 y 2 . Algunos de los parámetros tienen un significado físico notable, $v_{\max }$ representa la velocidad límite cuando la densidad es cercana a cero, $\rho_{\max }$ representa la densidad máxima que hace que la velocidad sea cercana a cero, $\rho_{c}$ representa el valor de la densidad cuando el flujo vehicular alcanza su valor máximo y $v_{c}$ es la velocidad cuando la densidad toma el valor de $\rho_{c}$. El encabezado ES hace referencia al error estándar.

Aunque todos los modelos modificados tienen buenos ajustes en general, es importante notar que muchos parámetros toman valores que no encajan con su significado físico, un ejemplo marcado de esto se ve en el modelo M7X donde $\rho_{\max }$ toma un valor demasiado alto, referirse a las Tablas 4,5 y 6 . 
Tabla 4: Información de los parámetros de los modelos asociada al carril 2.

\begin{tabular}{|c|c|c|c|c|c|c|c|c|c|}
\hline Modelos & $a$ & $b$ & $v_{\max }$ & $v_{c}$ & $\rho_{\max }$ & $\rho_{c}$ & $n$ & $m$ & ES \\
\hline M1 & & & 89.52 & & 87.12 & & & & 26.86 \\
\hline M1X & 44.03 & 17.51 & & & 231.38 & & & & 6.09 \\
\hline M2 & & & & 45.53 & 114.08 & & & & 27.1 \\
\hline M2X & 13.94 & 4.68 & & & 671.15 & & & & 5.19 \\
\hline M3 & & & 113.86 & & & 45.51 & & & 27.14 \\
\hline M3X & 52.6 & 19.14 & & & & 132.12 & & & 5.65 \\
\hline M4 & & & 70.61 & & & 45.36 & & & 26.94 \\
\hline M4X & 39 & 16.01 & & & & 102.28 & & & 6.55 \\
\hline M5 & & & 135 & & 96.5 & & & & 26.98 \\
\hline M5X & 59.5 & 22.05 & & & 304.47 & & & & 5.62 \\
\hline M6 & & & 98.1 & & 132.45 & & & & 27.02 \\
\hline M6X & 47.8 & 18.27 & & & 356.15 & & & & 5.88 \\
\hline M7 & & & 48.42 & & 61.41 & & 169.7 & 339.2 & 23.32 \\
\hline M7X & $5 \mathrm{e} 7$ & $1.5 \mathrm{e} 7$ & & & $9.1 \mathrm{e} 10$ & & 0.4 & $7.5 \mathrm{e} 9$ & 4.96 \\
\hline
\end{tabular}

Tabla 5: Información de los parámetros de los modelos, asociados al carril 4.

\begin{tabular}{|c|c|c|c|c|c|c|c|c|c|}
\hline Modelos & $a$ & $b$ & $v_{\max }$ & $v_{c}$ & $\rho_{\max }$ & $\rho_{c}$ & $n$ & $m$ & ES \\
\hline M1 & & & 91.01 & & 77.68 & & & & 16.12 \\
\hline M1X & 8.65 & 30.21 & & & 151.14 & & & & 4.45 \\
\hline M2 & & & & 53.32 & 89.82 & & & & 16.37 \\
\hline M2X & 3.03 & 11.57 & & & 253.33 & & & & 4 \\
\hline M3 & & & 147.2 & & & 32.15 & & & 16.56 \\
\hline M3X & 10.03 & 37.12 & & & & 77.07 & & & 3.97 \\
\hline M4 & & & 75.92 & & & 38 & & & 16.38 \\
\hline M4X & 7.55 & 27.54 & & & & 67.1 & & & 4.46 \\
\hline M5 & & & 144.59 & & 82.19 & & & & 16.23 \\
\hline M5X & 11.9 & 41.99 & & & 175.21 & & & & 4.11 \\
\hline M6 & & & 108.89 & & 108.43 & & & & 16.36 \\
\hline M6X & 9.41 & 33.38 & & & 219.16 & & & & 4.13 \\
\hline M7 & & & 69.2 & & 8.21 & & 18.29 & -2.08 & 20.46 \\
\hline M7X & 8.91 & 34.76 & & & $1.3 \mathrm{e} 5$ & & 1.54 & $1.5 \mathrm{e} 5$ & 3.91 \\
\hline
\end{tabular}


Tabla 6: Información de los parámetros de los modelos, asociados al carril 5.

\begin{tabular}{|c|c|c|c|c|c|c|c|c|c|}
\hline Modelos & $a$ & $b$ & $v_{\max }$ & $v_{c}$ & $\rho_{\max }$ & $\rho_{c}$ & $n$ & $m$ & $\mathrm{ES}$ \\
\hline M1 & & & 101.52 & & 68.52 & & & & 10.56 \\
\hline M1X & -3.7 & 37.85 & & & 140.59 & & & & 5.23 \\
\hline M2 & & & & 68.92 & 71.8 & & & & 10.65 \\
\hline $\mathrm{M} 2 \mathrm{X}$ & 4.14 & 14.88 & & & 182.49 & & & & 4.2 \\
\hline M3 & & & 250.12 & & & 21.39 & & & 11.33 \\
\hline M3X & 9.8 & 47.35 & & & & 58.49 & & & 4.03 \\
\hline M4 & & & 99.11 & & & 29.81 & & & 10.66 \\
\hline M4X & -3.34 & 36.73 & & & & 56.85 & & & 4.61 \\
\hline M5 & & & 170.33 & & 69.75 & & & & 10.54 \\
\hline M5X & 2.6 & 52.23 & & & 151.6 & & & & 4.56 \\
\hline M6 & & & 143.65 & & 84.97 & & & & 10.64 \\
\hline M6X & 1.61 & 42.72 & & & 183.45 & & & & 4.39 \\
\hline M7 & & & 56.98 & & 240.34 & & 7.59 & $2.3 \mathrm{e} 5$ & 9.9 \\
\hline M7X & 6.25 & 38.97 & & & $3.9 \mathrm{e} 4$ & & 1.98 & $4.9 \mathrm{e} 5$ & 3.92 \\
\hline
\end{tabular}

Para finalizar esta sección, se contrastarán los parámetros de los modelos, con las estimaciones hechas en la sección tres de este artículo. Para iniciar nótese que los modelos M3X y M4X son los que mejor ajustan el parámetro $\rho_{c}$ en comparación con los modelos M3 y M4. El parámetro estimado de la velocidad máxima se ve claramente más cercano a los modelos modificados, en comparación con los modelos clásicos. Por último se observa que las estimaciones de las densidades máximas encontradas en la sección 3 son más cercanas a los ajustes encontrados con los modelos clásicos.

\section{Conclusiones}

En este artículo se logró probar que los modelos modificados con una variación lineal en el espacio, se ajustan notablemente mejor que los modelos clásicos, se conjetura que esto puede ser debido a situaciones propias de la carretera y no del estado de la densidad. Particularmente se observó que el mejor modelo en conjunto es el modelo de Underwood modificado, siguiéndole en este sentido el modelo modificado de Drake.

Los modelos de Greenshields, Greenberg, Drew, Pipes y Nicholas modificados, presentan buenos ajustes, sin embargo, en algunos casos los parámetros involucrados pierden su sentido físico, hecho que se evidenció con las estimaciones encontradas en la sección 3. 
El autor de este artículo cree humildemente que este tipo de trabajos juegan un papel importante en la investigación aplicada al tráfico vehicular, dado que muchas veces la elección de un modelo forma parte de las bases de un proyecto de investigación. Una de las más notables aplicaciones de los modelos macroscópicos es su capacidad de modelar la dinámica del tráfico a gran escala, como un ejemplo claro de esto se citan los trabajos [5] y [2], donde se hace uso de los modelos clásicos.

Una de las principales características de este trabajo es que se ha logrado comparar diversos modelos con la incorporación de un término lineal en la variable espacial y se han podido contrastar con datos reales del tráfico.

\section{Agradecimientos}

El autor de este trabajo agradece de manera general a la carrera y la maestría de matemática de la Universidad Nacional Autónoma de Honduras por la invaluable formación en el Â rea. De forma particular se agradecen las oportunas observaciones hechas por los revisores anónimos asignados por esta revista; observaciones que sinceramente han enriquecido este trabajo..

\section{Fuentes de financiamiento}

Investigación parcialmente financiada por la Universidad Nacional Autónoma de Honduras y el autor.

\section{Referencias}

[1] J.M. Alvarenga-Rodríguez, Reconstrucción del proyecto NGSIM, Youtube. https://youtu.be/hln3F8TRmKY

[2] J. Buli, Y. Xing, A discontinuous Galerkin method for the aw-rascle traffic flow model on networks, Journal of Computational Physics 406(2020), article 109183. Doi: $10.1016 / j \cdot j c p .2019 .109183$

[3] J.L. Drake, J.L. Schofer, A statistical analysis of speed-density hypotheses, High Research Record 154(1966), 53-87.

[4] D.R. Drew, Traffic flow theory and control, McGraw-Hill, New York NY, 1968.

[5] P. Goatin, E. Rossi, A multiLane macroscopic traffic flow model for simple networks, SIAM Journal on Applied Mathematics 79(2019), no. 5, 1967-1989. Doi: 10.1137/19M1254386

Rev.Mate.Teor.Aplic. (ISSN print: 1409-2433; online: 2215-3373) Vol. 28(1): 125-142, Jan-Jun 2021 
[6] H. Greenberg, An analysis of traffic flow, Op res, 7(1959), no. 1, 79-85. Doi: 10.1287 /opre.7.1.79

[7] B.D. Greenshields, J.R. Bibbins, W.S. Channing, H.H. Miller, A study of traffic capacity, High Research Board Proc.14(1935), no. 1, 448-477.

[8] M.J. MacNicholas, A simple and pragmatic representation of traffic flow, in: Symp on the Fundamental Diagram: 75 Years (Greenshields 75 Symposium), Transportation Research Board, Woods Hole MA, United States, 2011. pp. 161-177.

[9] L.A. Pipes, Car following models and the fundamental diagram of road traffic, Transp Res 1(1967), no. 1, 21-29. Doi: 10.1016/ $0041-1647$ (67) 90092-5

[10] Transportation.gov, Next Generation Simulation (NGSIM) Vehicle Trajectories and Supporting Data, U.S. Department of transportation, 2017. https://data.transportation.gov/Automobiles/ Next-Generation-Simulation-NGSIM-Vehicle-Trajector/ $8 e c t-6 j q j$

[11] R.T. Underwood, Speed, Volume, and Density Relationships: Quality Theory of Traffic Flow. Yale University Bureau of Highway Traffic, University Park, PA, United States,1961, pp. 141-187.

[12] P. Zhang, R. Liu, Hyperbolic conservation laws with space-dependent flux: I. Characteristics theory and Riemann problem, Jou. Comp. App. Maths. 156(2003), no. 1, 1-21. Doi: 10 .1016/S0377-0427 (02) 00880-4 